\title{
Relieving compression against injured spinal cord via non-suturing muscle layer in rat.
}

\author{
Feng Zhao" ${ }^{1 \#}$, Xiao-Yan Ding ${ }^{2 \#}$, Feng Wu ${ }^{3}$, Xiao-Hui Li ${ }^{4}$, Yu-Huan Li ${ }^{5}$, Mei Hu ${ }^{6}$, Sheng-Li Huang ${ }^{*}$ \\ ${ }^{1}$ Department of Thoracic Surgery, the Second Affiliated Hospital, School of Medicine, Xi'an Jiaotong University, Xi'an, \\ PR China \\ ${ }^{2}$ Department of Ophthalmology, Xi'an No 3 Hospital, Xi'an, PR China \\ ${ }^{3}$ Department of Pathogenic Biology and Immunology, School of Medicine, Xi'an Jiaotong University, Xi'an, PR China \\ ${ }^{4}$ Departments of Radiology, the Second Affiliated Hospital, School of Medicine, Xi'an Jiaotong University, Xi'an, PR China \\ ${ }^{5}$ Department of Orthopaedics, the Second Affiliated Hospital, School of Medicine, Xi'an Jiaotong University, Xi'an, \\ PR China
}

${ }^{6}$ Editorial Office, Infectious Disease Information, Beijing, PR China

\#These two authors contributed equally to this study.

\begin{abstract}
Background: The sustained compression to the spinal cord caused by muscle scars after surgery in Spinal Cord Injury (SCI) models makes it difficult to evaluate the effectiveness of therapeutic regimens. The present study aims to evaluate potential strategies to reduce such compression.

Materials and methods: Rat SCI models were used and divided into two groups: one with muscle layer sutured after surgery and the other not sutured. The time point of muscle scar formation was determined by hematoxylin and eosin stain. Magnetic Resonance Image (MRI) was performed after the determined time point to observe the severity of compression of muscle scar against the injured spinal cord in both groups. The Basso, Beattie, and Bresnahan score (BBB score) was used to evaluate the locomotor outcomes.

Results: The scar formed in the third week after the muscle was dissected. MRI in the fourth week after surgery indicated that the rats with muscle layer sutured experienced more severe compression against the spinal cord than those not sutured. The concave caused by muscle scars in the injured spinal cord tissue of the muscle layer sutured rats was more obvious than that in non-sutured ones. There were no statistically significant differences in the BBB score between the two groups $(p>0.05)$.

Conclusion: The compression to the injured spinal cord caused by muscle scars after surgical procedures could be relieved if the muscle layer is not sutured, and this non-suturing management may makes it easy to evaluate the effectiveness of therapeutic regimens in rat SCI models.
\end{abstract}

Keywords: Spinal cord injury, Muscle scar formation, Compression, MRI, Rat.

Accepted on March 01, 2018

\section{Introduction}

Treatment and rehabilitation of Spinal Cord Injury (SCI) are still major challenges today [1-3]. Animal models of SCI are a necessary prerequisite for the evaluation of suitable therapeutic regimens clinically [4-6]. An ideal animal model should be able to reflect the process of pathological changes of the injury, and meet the requirements of availability and repeatability [7]. The model also could be used to evaluate the effectiveness of therapeutic regimens [8].
In most rat SCI procedures reported previously, the muscle layer was sutured after dissection $[4,9,10]$. We followed this management in our own experiments, but found that severe compression to injured spinal cord tissue was caused by the scars formed after muscle dissection. The compression might make any management invalid and make it difficult to evaluate the therapeutic effects of the treatment. In our later surgical procedures on SCI models, we noticed that the compression was reduced in animals without suturing muscle layer. 
To verify this observation, we conducted the present study to compare the compression resulted from the operation of muscle suturing as against the operation of non-suturing.

\section{Material and Methods}

\section{Animals}

Ethical approval for the study was obtained from the Ethics Committee of the Second Affiliated Hospital, School of Medicine, Xi'an Jiaotong University. Principles of laboratory animal care were followed, and all procedures were conducted according to guidelines established by the National Institutes of Health. A total of 10 male Sprague-Dawley (SD) rats $(250 \mathrm{~g} \pm$ $10 \mathrm{~g}$ ) were used in our experiments. The rats were divided into two groups $(n=5)$ randomly: the SCI models with the muscle layer sutured (sutured group) and those with the muscle layer not sutured (non-sutured group).

\section{Preparing SCI models}

The rat was anesthetized with an intraperitoneal injection of $10 \%$ chloral hydrate solution $(350 \mu \mathrm{l} / 100 \mathrm{~g})$. After the surgical field prepared and disinfected, the layer of skin, anadesma and muscle were opened respectively and a laminectomy was performed at the T10 level to expose the target spinal cord segment. The rat was then placed in an impactor (the NYU weight-drop device, New York University, New York, USA) and a $10 \mathrm{~g}$ rod was dropped from a height of $25 \mathrm{~mm}$ to cause SCI.

After that, the muscle, anadesma and skin were sutured in layers in the sutured group. For the non-sutured group, the incision of muscle was aligned but not sutured, and the anadesma and skin were sutured in layers. The rats were then placed on postoperative care with a bi-daily procedure, which involved bladder expression and intramuscular injection of one dose of penicillin (North China pharmaceutical Co., Ltd. China; 200,000 units per rat). The postoperative care procedure lasted until the rats regained bladder function and showed no signs of infection or stress.

\section{Determining the time point of muscle scar formation}

Muscle tissues were obtained from the surgical field in 7, 14, 21, $28 \mathrm{~d}$ after SCI procedures. The tissues were embedded in paraffin and sectioned at a thickness of $6 \mu \mathrm{m}$. Four slices of each specimen were randomly selected for hematoxylin and eosin ( $\mathrm{H}$ and $\mathrm{E})$ staining.

\section{Evaluating the postoperative compression to the spinal cord}

Magnetic Resonance Imaging (MRI) was performed after the determined time point of scar formation and gross morphology of the injured spinal cord was observed. Data on the damaged area were acquired on a 3T MR scanner (Signa; GE Medical Systems, Milwaukee, Wisconsin, USA) using a specialized coil designed for scanning rats. The rat was placed in the coil in a supine position to reduce the influence of respiratory movements on magnetic resonance artifacts of the spinal cord. The relevant parameters of T1-weighted and T2-weighted images of the injured rats were as follows: $\mathrm{TR}=560$ and 2600 $\mathrm{ms}$, respectively; $\mathrm{TE}=11.3$ and $120 \mathrm{~ms}$, respectively; matrix size $=320 \times 224, \mathrm{FOV}=8$, and slice thickness $=1.5 \mathrm{~mm}$ in both .

\section{Assessing locomotor function}

Hind limb locomotor function was analyzed using the Basso, Beattie and Bresnahan (BBB) open-field locomotor test $[11,12]$. The hind limb movements of all the injured rats were recorded every $3 \mathrm{~d}$ through video recording by one of the researchers. The video recording was performed at 8:00 p.m. because of the large difference in the circadian activity of rats between day-time and night-time. The locomotor BBB scores were evaluated by two other researchers independently.

\section{Statistical analysis}

BBB scores were expressed as mean \pm standard deviation (SD) and performed using the SPSS 18.0 software package. Student's ttest was performed for statistical evaluation. Pvalue $<0.05$ was considered statistically significant.

\section{Results}

\section{Time point of muscle scar formation}

The $\mathrm{H}$ and $\mathrm{E}$ staining of the muscle sections revealed granulation tissues in 7,14 d and scar tissues in the $21,28 \mathrm{~d}$ after the SCI procedures (Figure 1). This observation indicated that scar tissue formation occurred roughly at the third week after the surgical management.
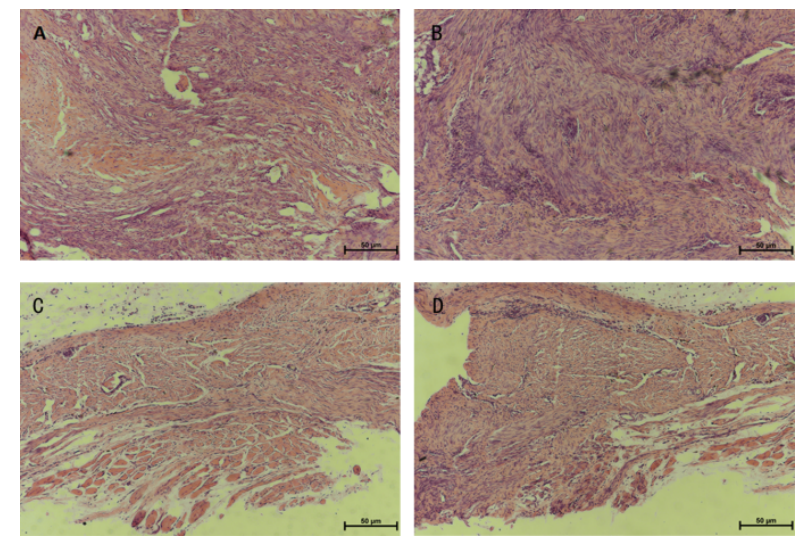

Figure 1. $H$ and E staining of muscle sections obtained from sutured muscle at different time points (X40). (A) $7 d$, granulation tissue; (B) $14 d$, granulation tissue; (C) $21 d$, scar tissue; (D) $28 d$, scar tissue.

\section{Postoperative compression to the spinal cord}

MRI data and gross morphology of injured spinal cord showed that the compression in rats with muscle layer sutured was notably more as compared to that in rats with muscle layer not sutured (Figures 2 and 3). Gross morphology of injured spinal cord obtained from the surgical field indicated that the concave 
caused by muscle scars in the injured spinal cord tissue of the muscle layer sutured rats was more obvious than that in nonsutured ones (Figure 3). These differences suggested that not suturing the muscle layer might reduce the compression caused by the scar tissue resulted from suturing the muscle.
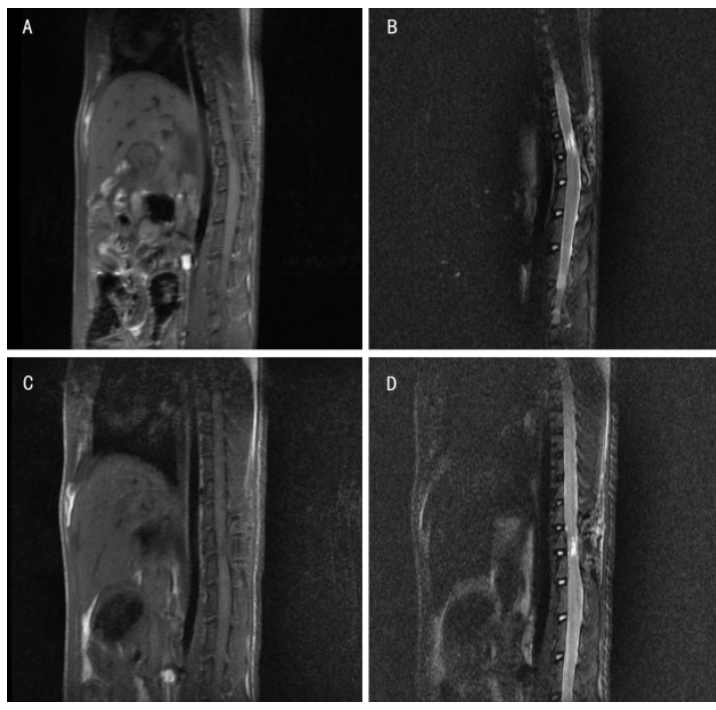

Figure 2. MRI of the SCI rats with the muscle layer sutured or not sutured at the fourth week after the SCI procedures. (A, B) T1weighted and T2-weighted images of a SCI rat with the muscle layer not sutured; an interval was between the injured spinal cord and posterior soft tissue; $(C, D)$ T1-weighted and T2-weighted images of a SCI rat with the muscle layer sutured; the compression to the injured spinal cord was caused by soft tissue.
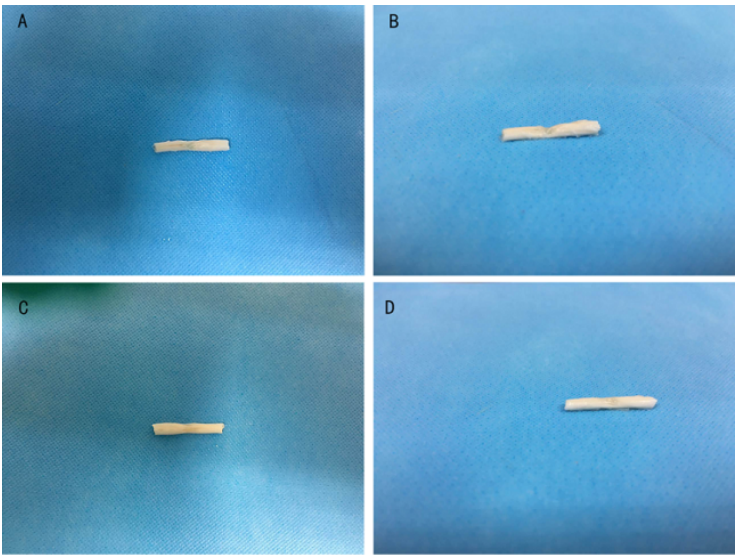

Figure 3. Frontal and profile images of the gross morphology of injured spinal cord tissue from SCI rats with the muscle layer sutured or not sutured. (A, B) The injured spinal cord was compressed severely in the sutured model; $(C, D)$ No severe compression to the injured spinal cord was seen in the non-sutured model.

\section{Locomotor function}

No significant differences $(\mathrm{P}>0.05)$ of the $\mathrm{BBB}$ scores were found between the two groups at different time points (Figure $4)$.

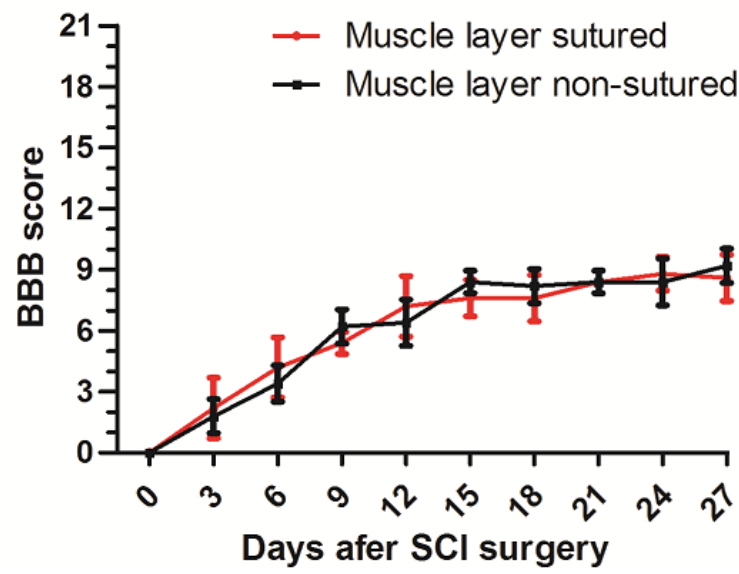

Figure 4. No obvious differences in BBB scores were observed between the two groups at each time point after the SCI procedures $(P>0.05)$.

\section{Discussion}

The present study evaluates a potential way to reduce the postsurgical compression to the injured spinal cord in rat SCI model. Our comparison between rats with the muscle layer sutured after SCI procedures and those with the muscle layer not sutured shows that the non-suturing operation resulted in notably less compression to the injured spinal cord. The MRI and morphological results demonstrate a relief of the compression in rats with the muscle layer not sutured. Meanwhile, the two different operations did not result in remarkable differences in the hind limb locomotor function, indicating that the decompression management would not cause instability in the rat SCI models. In brief, our experimental results suggest a potential way to reduce the compression to the injured spinal cord caused by surgical procedures in SCI animal models. It may offer some novel ideas for clinical practices, such as laminectomy.

Evaluation of any therapy for SCI requires the use of animal models. For years, Allen's collision model has been widely used $[9,10,13]$. However, little attention has been paid to how to improve the models. A severe compression to the spinal cord will certainly worsen the injury, which may hinder objective evaluation of the effects of therapeutic regimens. Animal experiments are the foundation of clinical managements of the injury, and an ideal animal model may accelerate the progress of relevant research [8]. The scar tissue as a result of muscle suturing may form sustaining compression to the injured spinal cord, due to which the therapeutic regimen under investigation may appear ineffective. This might be a crucial reason for the slow development in treatment and rehabilitation of SCI in recent years. We believe that reliving the compression in SCI animal models would improve this situation.

There are some limitations in this study. The study just offered a method to make a curable rat SCI model, which need further verified by therapeutic regimens. Because spinal cord of the rat is too small and the axial image of MRI is unrecognizable, the 
quantitative data, such as the amount of compression on axial sections was difficult to obtain. Therefore, we did not compare the data of the axial images of MRI between two groups. In addition, electrophysiology recording should be utilized in assessing a wide variety of spinal procedures, especially after SCI.

\section{Conclusion}

Not-suturing the muscle layer in a rat SCI model would relieve the compression to the injured spinal cord caused by the scar tissue after the SCI procedure. This operation helps improve the animal model for SCI, which increases the potential of therapeutic methods under concern to cure the injury, and enables more accurate evaluation of the effectiveness of the therapeutic regimens. Hopefully, this may help locate better clinical management strategies for laminectomy.

\section{Acknowledgement}

This study was supported by research grants from the National Natural Science Foundation of China (No. 81271340 and No. 81471247) and Science and Technology Project of Xi'an, China (No.2016048SF/YX04 (3)).

\section{References}

1. Moonen G, Satkunendrarajah K, Wilcox JT, Badner A, Mothe A, Foltz W, Fehlings MG, Tator $\mathrm{CH}$. A new acute impact-compression lumbar spinal cord injury model in the rodent. J Neurotrauma 2016; 33: 278-289.

2. Wang J, Pearse D. Therapeutic hypothermia in spinal cord injury: the status of its use and open questions. Int $\mathrm{J}$ Mol Sci 2015; 16: 16848-16879.

3. Van Goethem JWM, Maes M, Özsarlak Ö, van den Hauwe L, Parizel PM. Imaging in spinal trauma. Eur Radiol 2005; 15: 582-590.

4. Wang F, Huang SL, He XJ, Li XH. Determination of the ideal rat model for spinal cord injury by diffusion tensor imaging. Neuroreport 2014; 25: 1386-1392.

5. Li XH, Wu F, Zhao F, Huang SL. Fractional anisotropy is a marker in early-stage spinal cord injury. Brain Res 2017; 1672: 44-49.
6. Li XH, Li JB, He XJ, Wang F, Huang SL, Bai ZL. Timing of diffusion tensor imaging in the acute spinal cord injury of rats. Sci Rep 2015; 5: 12639.

7. Wang Y, Liu C, Wang Q, Gao H, Na H, Yu R. Establishment of a spinal cord injury model in adult rats by an electrocircuit-controlled impacting device and its pathological observations. Cell Biochem Biophys 2014; 69: 333-340.

8. Kjell J, Olson L. Rat models of spinal cord injury: from pathology to potential therapies. Dis Model Mech 2016; 9: 1125-1137.

9. Kanekiyo K, Nakano N, Noda T, Yamada Y, Suzuki Y, Ohta M, Yokota A, Fukushima M, Ide C. Transplantation of choroid plexus epithelial cells into contusion-injured spinal cord of rats. Restor Neurol Neurosci 2016; 34: 347-366.

10. Liu D, Hua J, Dong QR, Sun YM, Gan MF. X-ray therapy promotes structural regeneration after spinal cord injury in a rat model. J Orthop Surg Res 2016; 11: 1-5.

11. Basso DM, Beattie MS, Bresnahan JC. Graded histological and locomotor outcomes after spinal cord contusion using the NYU weight-drop device versus transection. Exp Neurol 1996; 139: 244-256.

12. Basso DM, Beattie MS, Bresnahan JC. A sensitive and reliable locomotor rating scale for open field testing in rats. J Neurotrauma 1995; 12: 1-21.

13. Ide C, Kanekiyo K. Points regarding cell transplantation for the treatment of spinal cord injury. Neural Regen Res 2016; 11: 1046-1049.

\section{*Correspondence to}

Sheng-Li Huang

Department of Orthopaedics

The Second Affiliated Hospital

School of Medicine

Xi'an Jiaotong University

Xi'an

PR China 